PHYSICAL REVIEW D 96, 059902(E) (2017)

\title{
Erratum: Electric conductivity of a hot hadron gas from a kinetic approach [Phys. Rev. D 93, 096012 (2016)]
}

\author{
Moritz Greif, Carsten Greiner, and Gabriel S. Denicol \\ (Received 22 August 2017; published 18 September 2017)
}

DOI: 10.1103/PhysRevD.96.059902

An error was found in the labeling of hadronic cross sections, shown in Fig. 3(b) of this paper. The corrected calculations are shown here in the left panel of Fig. 1 and display minor differences from the original results.

In the paper, Fig. 4 shows the result for all 11 species and the full set of cross sections in Table I. We argued that the dip at about $T=100 \mathrm{MeV}$ came from resonance peaks in the cross sections; however, it was found that it was due to a small numerical error. We show the corrected result in the right panel of Fig. 1 (red solid curve). We emphasize that no qualitative differences exist and the numerical errors did not affect any of the conclusions reached in this paper.

Note that in the right panel of Fig. 1 we changed the unit of the temperature axis from $\mathrm{GeV}$ to the critical temperature $T_{c}$ to better compare to other results (e.g., lattice), which are sometimes exclusively given in units of a critical temperature. (For the calculations that do not have an intrinsic critical temperature, like ours, we use $T_{c}=160 \mathrm{MeV}$.) The lattice QCD results are from Refs. [1-3], and all other references are given in the original publication. Note that we only compute a hadron resonance gas, and all results above $T=T_{c}$ are merely to show how our hadronic system would extrapolate at higher temperatures.
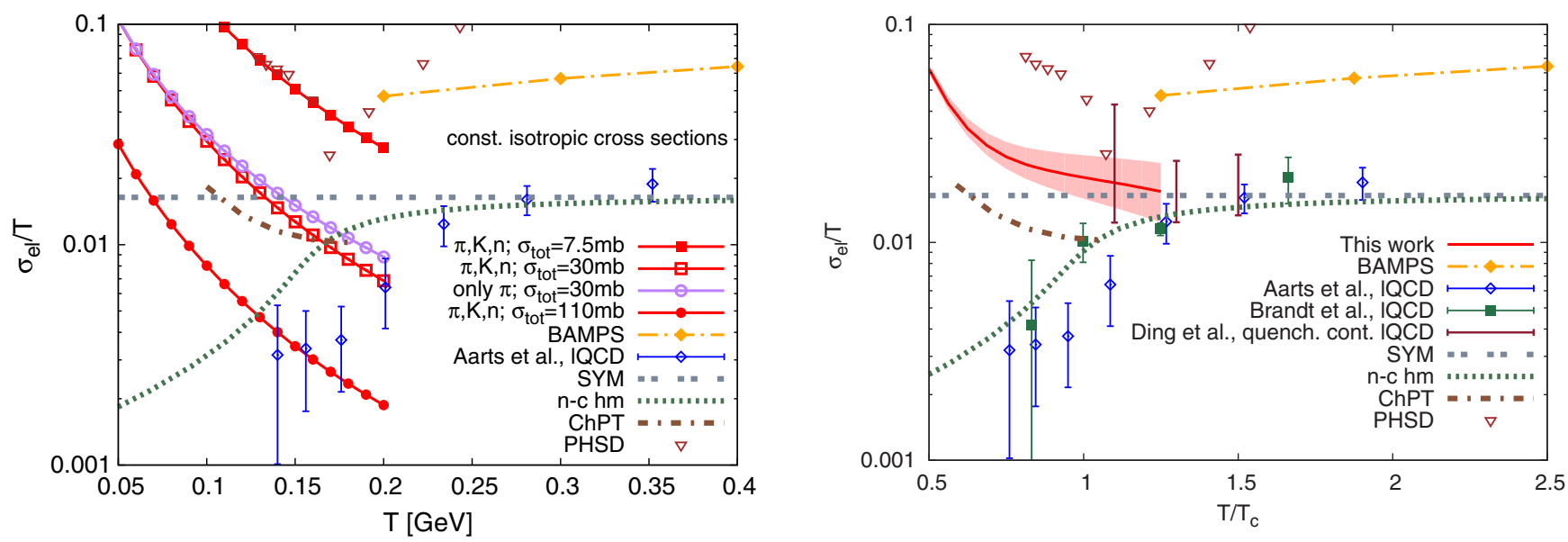

FIG. 1. Electric conductivity over temperature for constant isotropic cross sections (left panel) and resonance cross sections (right panel). The red band shows the uncertainty in those cross sections, which we approximated as constant and which we vary by a factor of $0.5-2$.

[1] G. Aarts, C. Allton, A. Amato, P. Giudice, S. Hands, and J.-I. Skullerud, J. High Energy Phys. 02 (2015) 186.

[2] B. B. Brandt, A. Francis, B. Jger, and H. B. Meyer, Phys. Rev. D 93, 054510 (2016).

[3] H.-T. Ding, O. Kaczmarek, and F. Meyer, Phys. Rev. D 94, 034504 (2016). 